
EFLUENTE DE INDÚSTRIA DE PESCADO

\title{
MEMBRANE TECHNOLOGYFOR THE TREATMENT OF FISH INDUSTRY EFFLUENT
}

\author{
PONSANO, Elisa Helena Giglio ${ }^{1 *}$; PIRES,Helenice Aparecida ${ }^{2}$; OLIVEIRA, Dayse Lícia ${ }^{3}$; \\ GARCIA, Andréa Fontes ${ }^{4}$ \\ 1, 2, 3 UnespUniv Estadual Paulista, Faculdade de Medicina Veterinária de Araçatuba, Departamento de Apoio, \\ Produção e Saúde Animal, Rua Clóvis Pestana, 793, CEP 16050-680, Araçatuba - SP, Brasil \\ (fone: +55183636 1381) \\ ${ }^{4}$ UniSalesiano Centro Universitário Católico Salesiano Auxilium, Rodovia Senador Teotônio Vilela 3821, CEP \\ 16016-500, Araçatuba - SP, Brasil. (fone: +55 18 36365252) \\ * Autor correspondente \\ e-mail: elisahgp@fmva.unesp.br
}

Received 5 December 2017; received in revised form 06 May 2018; accepted 07 May 2018

\section{RESUMO}

A filtração por membranas desponta como uma alternativa para o tratamento de efluente de processamento de pescado. O objetivo deste trabalho foi verificar a capacidade da filtração por membranas em reduzir a carga poluente do efluente de processamento de tilápias. As filtrações foram realizadas com membranas de 150, 30 e $10 \mathrm{kDa}$. Os parâmetros fisicoquímicos do efluente in natura e dos permeados foram comparados entre si e com os padrões de lançamento previstos na legislação brasileira para avaliar a possibilidade de descarte direto em corpos d'água. As três membranas apresentaram o mesmo potencial para remover sólidos totais, nitrogênio e nitrito do efluente. As membranas de 30 e $10 \mathrm{kDa}$ provocaram efeitos semelhantes quanto à remoção da Demanda Química de Oxigênio e de proteínas. Os parâmetros óleos e graxas, $\mathrm{pH}$ e sólidos fixos não se alteraram com as filtrações. Todas as membranas foram eficazes na redução da cor do efluente. O efluente in natura já atendia aos padrões de lançamento quanto a temperatura, $\mathrm{pH}$, nitrogênio total e nitrito e o uso das membranas permitiu o atendimento aos padrões para materiais flutuantes. A cor e o teor de óleos e graxas dos permeados esteve acima do permitido para o lançamento em águas doces, sugerindo a utilização de operações adicionais para o atendimento à legislação.

Palavras-chave: demanda química de oxigênio, proteínas, sólidos totais, ultrafiltração

\section{ABSTRACT}

Membrane filtration emerges as an alternative for the treatment of fish processing effluent. The aim of this work was to verify the ability of membrane filtration on reducing the pollutant load of tilapia processing effluent. The filtrations were performed with 150, 30 and $10 \mathrm{kDa}$ membranes. The physicochemical parameters of the effluent in natura and the permeates were compared among themselves and with the standards for launching foreseen in the Brazilian legislation to evaluate the possibility of direct disposal in water bodies. The three membranes had the same potential to remove total solids, nitrogen and nitrite from the effluent. Membranes 30 and $10 \mathrm{kDa}$ caused similar effects on the removal of Chemical Oxygen Demand and proteins. Oils and greases, $\mathrm{pH}$ and fixed solids did not change with the filtrations. All the membranes were effective in reducing the color of the effluent. The effluent in natura was already in agreement with the standards for discharge regarding to temperature, $\mathrm{pH}$, total nitrogen and nitrite, and the use of the membranes allowed it to meet the standards for floating materials. The color and the content of oils and grease in permeates were above the levels allowed for the discharge in freshwater, so suggesting the use of an additional operation to comply with the legislation.

Keywords: chemical oxygen demand, proteins, total solids, ultrafiltration. 


\section{INTRODUÇÃO}

A indústria de alimentos é a que apresenta, dentre todas, o maior consumo de água, já que ela é utilizada em várias etapas dos processos produtivos industriais (Casani et al., 2005). A indústria de pescado, por exemplo, consome grandes quantidades de água em operações como limpeza, abate, descabeçamento e evisceração, filetagem, lavagem, resfriamento, descongelamento, acondicionamento, etc. (Cristovão et al., 2012).

No caso de indústria de enlatados, etapas adicionais também requerem água, tais como salga, enlatamento, cozimento, lavagem de latas e autoclavagem. Além disso, a água também é consumida para a lavagem de chão, utensílios e equipamentos (Rodrigues et al., 2017).

Após utilizadas, essas águas originam as águas residuárias, ou efluentes, que apresentam características distintas, de acordo com o volume e o tipo de processamento industrial (Giordano, 2004). Uma indústria de processamento de pescado com produção média de $100 \mathrm{t}$ por hora, por exemplo, pode produzir entre 20.000 a $40.000 \mathrm{~L}$ de efluente por hora, com parâmetros físicos e químicos variáveis (Afonso; Borquez, 2002a).

A caracterização fisicoquímica do efluente é importante para saber se ele atende aos padrões regulamentares para o lançamento em corpos d'água ou para reuso e/ou reciclagem e, no caso do não atendimento, ele deve receber tratamento até atingir adequação ao destino (Rodrigues et al., 2017). O tratamento dos efluentes industriais, portanto, se justifica por razões econômicas e ambientais (Cristovão et al., 2012).

A produção global de pescado tem aumentado nas últimas décadas, o que vem ao encontro da segurança alimentar para uma população estimada em 9 bilhões de pessoas em 2050 (FAO, 2016), uma vez que o pescado representa uma fonte proteica de boa qualidade e com preços acessíveis para a população mais vulnerável (FAO, 2013).

Em 2014, a produção mundial de pescado atingiu 167,2 milhões de toneladas, com destaque para a produção por aquacultura, em crescimento vertiginoso. Naquele mesmo ano, o Brasil figurava como $14^{\circ}$ produtor mundial de pescado nessa modalidade (FAO, 2016).
Dados nacionais apontam a tilápia como a espécie mais cultivada em pisciculturas de águas continentais no Brasil (Brasil, 2012), devido a características próprias da espécie, tais como facilidade de reprodução, excelente capacidade de crescimento em diferentes condições e resistência a doenças, além da boa aceitação do mercado consumidor (FAO, 2014).

Porém, em consequência da produção crescente de pescado, o setor de beneficiamento também vem crescendo e gerando grandes quantidades de efluentes para os quais o tratamento é particularmente difícil devido ao alto conteúdo de matéria orgânica e de sais que possuem (Cristovão et al., 2012). O tratamento convencional dos efluentes é realizado por coagulação e flotação por ar dissolvido. Entretanto, esses processos têm perdido espaço, principalmente por apresentarem o inconveniente da grande quantidade de lodo produzido, o que demanda custos adicionais para o descarte correto (Kuca; Szaniawska, 2009).

A tecnologia de membranas é um processo utilizado para separar substâncias dissolvidas e partículas finas de soluções. A membrana age como uma barreira semipermeável e seletiva, que separa as partículas com base em seu peso molecular e é caracterizada pelo tamanho de seu poro ou pelo peso molecular da partícula que retém (chamado peso de corte, expresso em KDa), originando os diferentes tipos de filtração: microfiltração (100 $500 \mathrm{KDa})$, ultrafiltração $(20-150 \mathrm{KDa})$, nanofiltração $(2-20 \mathrm{KDa})$ e osmose reversa $(0,2$ - $2 \mathrm{KDa}$ ) (Muro et al., 2012).

A filtração por membranas é dirigida por pressão e pode ser conduzida de duas formas distintas: filtração frontal (dead-end ou convencional) e filtração em fluxo cruzado ou tangencial (cross-flow) (Habert et al., 2006) (Figura 1). A filtração tangencial apresenta vantagens sobre a frontal, tais como menor entupimento e constância de fluxo e, por isso, é a mais utilizada industrialmente (Ghiggi, 2011; Shriniwass; Sharma, 2010).

A tecnologia de membranas para 0 tratamento de efluentes vem encontrando uso crescente na indústria do pescado, em particular a ultrafiltração e a nanofiltração. As vantagens dessa técnica sobre os processos convencionais são a boa qualidade do permeado obtido, que pode ser reciclado na própria planta de processamento, além da possibilidade simultânea de recuperação das proteínas 
concentradas no retentado (Afonso et al., 2004).

O uso da tecnologia de membranas para o tratamento de efluentes da indústria de pescado permite a recuperação de uma série de compostos valiosos tais como proteínas, enzimas e colágeno e, assim, não apenas reduz o risco de poluição ambiental mas, também, incrementa os benefícios econômicos para a indústria (Shriniwass; Sharma, 2010)

Considerando o cenário favorável para a produção de pescado em nível mundial e nacional, o uso da filtração por membranas pode representar uma alternativa para o tratamento dos efluentes provenientes da industrialização. $O$ objetivo deste trabalho foi testar a aplicação da filtração por membranas para a redução da matéria orgânica de efluente de processamento de tilápias.

\section{MATERIAL E MÉTODOS}

\subsection{Efluente}

O efluente utilizado no experimento foi obtido de indústria de abate, filetagem e congelamento de tilápias (Oreochromis niloticus), localizada no município de Aparecida do Taboado - MS, com produção média estimada em 30 mil toneladas de peixe por ano. As amostras foram coletadas do tanque de lavagem, operação prévia ao congelamento (Figura 2).

\subsection{Caracterização Físicoquímica do Efluente In Natura e dos Permeados}

Sólidos totais, sólidos fixos, óleos e graxas, proteínas e $\mathrm{pH}$ foram quantificados conforme as técnicas descritas em APHA et al. (2005). Nitrogênio total e nitrito foram determinados por métodos colorimétricos (métodos Hach 8075, 8038 e 8507, respectivamente). A cor foi determinada em espectrofotômetro (método Hach 8025). A demanda química de oxigênio (DQO) foi determinada segundo a metodologia de Jirka; Carter (1975), com adaptações, em que a digestão química foi realizada em reator (Hach DRB - 200) e a análise colorimétrica foi realizada em espectrofotômetro (Hach DR - 2800), a partir de curva de calibração apropriada.

\subsection{Tratamentos}

O efluente in natura foi submetido a uma filtração prévia em tecido musseline (160 fios/polegadas) para a retenção de partículas grosseiras. Em seguida, foram realizadas as filtrações tangenciais em circuito fechado, utilizando um sistema constituído de tanque de recepção e concentração, bomba hidráulica, adaptador para membrana e manômetro (Figura 3). Foram testados três módulos de membrana: M1 - $150 \mathrm{kDa}, \mathrm{M} 2$ - $30 \mathrm{kDa}$ e M3 - $10 \mathrm{kDa}$ (Microdyn-Nadir). A membrana de $150 \mathrm{kDa}$ era constituída de acetato de celulose e as membranas de poros menores (30 e $10 \mathrm{kDa}$ ) eram constituídas de polietersulfona. As filtrações foram realizadas em temperatura ambiente, partindo de $50 \mathrm{~L}$ e com recirculação do retentado, até a obtenção de $8 \%$ do volume inicial

Dessa forma, o experimento contou com quatro tratamentos. Um deles correspondeu ao efluente sem qualquer filtração (EN) e os outros três corresponderam ao efluente filtrado com as membranas $\mathrm{M} 1, \mathrm{M} 2$ e $\mathrm{M} 3$. Foram realizadas três repetições de cada filtração.

A velocidade de escoamento foi calculada pela medição do permeado gerado em cada filtração dentro de um determinado período de tempo. A eficiência das membranas em remover material poluente do efluente foi expressa em \% de rejeição, conforme sugerido por Afonso et al. (2004), utilizando a Equação 1.

\subsection{Análise de Conformidade com a Legislação para o Descarte Direto}

Para a análise de possibilidade de descarte direto em corpos d'água, os valores obtidos nas análises físico-químicas do efluente in natura e dos permeados foram comparados com os padrões de lançamento previstos pelo Conselho Nacional do Meio Ambiente, CONAMA (Brasil, 2011).

\subsection{Análise Estatística}

Os resultados dos parâmetros físicoquímicas do efluente in natura e do permeado de cada filtração foram submetidos à análise de variância, seguida do teste de Tukey para a comparação das médias, duas a duas. O programa utilizado foi o software Action Versão 2.9 e adotou-se o nível de significância de 5\%. 


\section{RESULTADOS E DISCUSSÃO}

As três membranas apresentaram 0 mesmo potencial para remover sólidos totais, nitrogênio e nitrito do efluente da indústria de processamento de tilápias (Tabela 1). No entanto, os valores de $\mathrm{pH}$, óleos e graxas e sólidos fixos não diferiram entre os tratamentos ( $p>0,05)$. Os tratamentos realizados com as membranas de 30 e $10 \mathrm{kDa}$ resultaram em efeitos semelhantes quanto à remoção de $\mathrm{DQO} e$ de proteínas do efluente. Os resultados obtidos para a recuperação de proteínas foram próximos aos alcançados por Afonso et al. (2004), que obtiveram $62 \%$ de rejeição utilizando membrana de $15 \mathrm{kDa}$ para filtrar efluente de processamento de peixe.

Todos os tratamentos foram eficazes na redução da cor do efluente, sendo que a membrana de $10 \mathrm{kDa}$ apresentou maior porcentagem de rejeição, 91,17\%, deixando o permeado com a cor aparente muito próxima da permitida para o lançamento em águas doces, que é de $75 \mathrm{mg} \mathrm{Pt/L}$ (Figura 4) (Brasil, 1986).

As velocidades de escoamento foram, respectivamente, $2 \mathrm{~L} / \mathrm{min}, 0,6 \mathrm{~L} / \mathrm{min}$ e $0,07 \mathrm{~L} / \mathrm{min}$ para os tratamentos com as membranas de 150 , 30 e $10 \mathrm{kDa}$. Considerando que as porcentagens de rejeição de proteínas, sólidos totais, DQO e cor não diferiram entre T2 e T3, sugere-se o uso da membrana de $30 \mathrm{kDa}$ para promover a redução de matéria orgânica do efluente resultante do processamento industrial de tilápias, já que, nessa porosidade, ela apresenta maior velocidade de escoamento e menor ocorrência de entupimentos que a membrana de $10 \mathrm{kDa}$. Apesar de não sofrer entupimento e apresentar uma filtração mais rápida, a rejeição de proteínas e de DQO alcançada com a membrana de 150 kDa (T1) não foi significativa $(p>0,05 \%)$.

Um problema encontrado no uso da tecnologia de membranas é o entupimento, que diminui a velocidade de escoamento e requer operações de limpeza frequentes, o que pode dificultar seu uso em escala industrial em função do aumento no tempo de tratamento e na mão de obra. No entanto, esse não deve ser considerado um impeditivo para o uso da técnica, uma vez que um ciclo de limpeza ácido/base é capaz de recuperar a permeabilidade das membranas (Afonso; Borquez, 2002b; Afonso et al., 2004) e pode ser planejado durante o tratamento, conforme realizado neste estudo.

De acordo com a Resolução CONAMA no 430 (Brasil, 2011), os efluentes de qualquer fonte poluidora somente poderão ser lançados diretamente nos corpos receptores desde que obedeçam a algumas condições e padrões. $O$ efluente in natura já se enquadrava na legislação quanto a temperatura (inferior a $40^{\circ} \mathrm{C}$ ), $\mathrm{pH}$ (entre 5 a 9), nitrogênio total (até $1,27 \mathrm{mg} / \mathrm{L}$ ) e nitrito (até $1 \mathrm{mg} / \mathrm{L}$ ). O uso de filtração por membranas permitiu o atendimento aos padrões de materiais flutuantes, porém, o teor de óleos e graxas resultantes em todos os tratamentos ainda esteve acima do permitido para o lançamento do permeado em águas doces (até $0,05 \mathrm{~g} / \mathrm{L}$ ).

Cristovão et al. (2014) testaram o uso das operações de sedimentação, coagulação/floculação e flotação por ar dissolvido no tratamento primário de efluente industrial de pescado enlatado e concluíram que a sedimentação por $1,5 \mathrm{~h}$ foi suficiente para atingir as máximas remoções de óleos e graxas e sólidos totais. Portanto, uma possibilidade para aumentar a remoção de óleos e graxas do efluente estudado, de modo a fazê-lo alcançar o padrão para lançamento é combinar a tecnologia de membranas com operações preliminares tais como sedimentação ou flotação. Essas operações, além de serem simples e eficientes, não requerem a utilização de produtos químicos, permitindo 0 aproveitamento das substâncias recuperadas, por exemplo, em formulações de ração animal (González, 1996). A combinação da tecnologia de membranas para o tratamento de efluentes de indústria de pescado com outros processos físicos ou bioquímicos permite a obtenção de produtos valiosos com alto grau de pureza e pode até mesmo alcançar a obtenção de água para reciclagem (Shriniwass; Sharma, 2010).

Outra possibilidade para aumentar a eficiência da remoção da carga poluente do efluente da indústria de pescado é combinar filtrações com membranas de porosidades decrescentes, como fizeram Afonso; Borquez (2002b), que utilizaram microfiltração seguida de nanofiltração para tratar o efluente de indústria de farelo de peixe. De acordo com Muro et al. (2012), um pré-tratamento bem escolhido e a seleção adequada da configuração da membrana 
são fundamentais para maximizar a remoção de substâncias dos resíduos industriais.

Novas investigações deverão ser conduzidas focando 0 uso de operações adicionais ou combinações de membranas de porosidades diferentes com o objetivo de melhorar a remoção da carga poluente do efluente de indústria de processamento de tilápias e, mais além, buscando a recuperação de compostos de interesse econômico, tais como peptídeos com atividades biológicas.

\section{CONCLUSÕES}

As filtrações com membranas de 10 e 30 $\mathrm{kDa}$ reduziram as porcentagens de proteínas, sólidos totais, DQO e cor do efluente de processamento de pescado, porém, a cor e o teor de óleos e graxas dos permeados resultantes ficaram acima do permitido para 0 descarte.

\section{AGRADECIMENTOS}

À FAPESP (Processo 2015/25853-1) pelo auxílio financeiro e à CAPES pela bolsa de mestrado.

\section{REFERÊNCIAS}

1. APHA, AWWA, WPCF. Standard Methods for the Examination of Water and Wastewater. $21^{\text {st }}$ Ed. American Public Health Association, Washington, 2005.

2. Afonso, M.D., Borquez, R. Desalination, 2002, 142, 29. a

3. Afonso, M.D., Borquez, R. Desalination, 2002, 151, 131. b

4. Afonso, M.D., Ferrer, J., Borquez, R. Trends Food Sci. Technol., 2004, 15, 506.

5. BRASIL. Ministério da Pesca e Aquicultura. Boletim estatístico da pesca e aquicultura: Brasil 2010. Brasília: Ministério da Pesca e Aquicultura, 2012.

6. BRASIL. Resolução CONAMA no 430 , de 13 de maio de 2011. Conselho Nacional do Meio Ambiente: CONAMA, Brasília, DF, maio de 2011.

7. BRASIL. Resolução CONAMA n 20 , de 18 de junho de 2011. Conselho Nacional do Meio Ambiente: CONAMA, Brasília, DF, junho de 1986.

8. Casani, S., Rouhany, M., Knøchel, S. Water Res., 2005, 39, 1134.
9. Cristovão, R.O. Botelho, C.M.S., Martins, R.J.E., Boaventura, R.A.R. Int. J.Biosci. Biochem. Bioinforma., 2012, 2, 237.

10. Cristovão, R.O. Botelho, C.M.S., Martins, R.J.E., Loureiro, J. M.; Boaventura, R.A.R. Water Resour Ind., 2014, 6, 51.

11. FAO. Food and Agriculture Organization of the United Nations. The State of world fisheries and aquaculture: contributing to food security and nutrition for alcontributing to food security and nutrition for all. Roma: FAO, 2016.

12. FAO. Food and Agriculture Organization of the United Nations. The state of world fisheries and aquaculture. Opportunities and challenges. Part one - World review of fisheries and aquaculture. Roma: FAO, 2014.

13. FAO. Food and Agriculture Organization of the United Nations. Incrementar 0 consumo e o comércio inter-regional de pescado pode contribuir para a luta contra a fome na América Latina e Caribe. Roma: FAO, 2013.

14. Giordano, G. Tratamento e controle de efluentes industriais. http://www.ufmt.br/esa/Modulo II Efluent es Industriais/Apost El 2004

1ABES Mato Grosso UFMT2. pdf.

15. González, J. P. Wastewater treatment in the fishery industry. FAO Fisheries technical paper - 355. Roma: FAO, 1996.

16. Ghiggi, F.F. Tratamento de águas para consumo domestico com membranas de ultrafiltração. Trabalho de Conclusão de Curso - Departamento de Engenharia Química. Escola de Engenharia. Universidade Federal do Rio Grande do Sul. Porto Alegre. 2011

17. Habert, A.C.; Borges, C.P.; Nobrega, R. Processos de separação por membranas. Rio de Janeiro: E-papers. 2006.

18. Jirka, A.M., Carter, M.J., Anal. Chem.,1975, 47, 1397.

19. Kuca, M.; Szaniawska, D. Desalination, 2009, 241, 227.

20. Muro, C.; Riera, F.; Díaz, M. C. Membrane separation process in wastewater treatment of food industry. In: Valdez, B. (Ed.). Food Industrial Processes - methods and equipment. Rijeka: Intech. 2012. 253.

21. Rodrigues, L. M.; Ferraciolli, V. D.; Luiz, D. B.; Naval, L. P. Rev. Ambient. Água, 
2017, 12, 730.

22. Shriniwas, B. D.; Sharma, R. Clean

Technol., 2010, 368. 


\section{Equação 1}

Rejeição (\%) $=\left(1-\frac{\text { Concentração do componente no permeado }}{\text { Concentração do componente no efluente in natura }}\right) \times 100$

Tabela 1 - Médias e desvios padrões dos parâmetros físico-químicos do efluente in natura (EN) e do permeado resultante das filtrações (M1, M2 e M3) e suas respectivas porcentagens de rejeição

\begin{tabular}{|c|c|c|c|c|}
\hline Parâmetro & EN & M1 & M2 & M3 \\
\hline $\mathrm{pH}$ & $7,27 \pm 0,42$ & $7,02 \pm 0,51$ & $6,95 \pm 0,77$ & $7,62 \pm 0,93$ \\
\hline $\begin{array}{l}\text { Nitrogênio Total } \\
\text { (ma/L) }\end{array}$ & $1,06 \pm 0,03^{\mathrm{a}}$ & $\begin{array}{c}0,134 \pm 0,035^{b} \\
(87,32)\end{array}$ & $\begin{array}{c}0,122 \pm 0,025^{b} \\
(87,48)\end{array}$ & $\begin{array}{c}0,131 \pm 0,043^{b} \\
(87,54)\end{array}$ \\
\hline Proteína (\%) & $0,26 \pm 0,06^{a}$ & $\begin{array}{c}0,18 \pm 0,05^{\mathrm{ab}} \\
(31.08)\end{array}$ & $\begin{array}{c}0,11 \pm 0,02^{b} \\
(56,28)\end{array}$ & $\begin{array}{c}0,12 \pm 0,04^{\mathrm{b}} \\
(51.81)\end{array}$ \\
\hline Óleos e graxas (g/L) & $0,46 \pm 0,26$ & $\begin{array}{c}0,31 \pm 0,02 \\
(32,33)\end{array}$ & $\begin{array}{c}0,23 \pm 0,17 \\
(49,46)\end{array}$ & $\begin{array}{c}0,19 \pm 0,13 \\
(59,93)\end{array}$ \\
\hline Sólidos fixos (g/L) & $1,18 \pm 0,21$ & $\begin{array}{c}1,06 \pm 0,05 \\
(9,77)\end{array}$ & $\begin{array}{c}1,00 \pm 0,12 \\
(15,21)\end{array}$ & $\begin{array}{c}0,97 \pm 0,16 \\
(17,62)\end{array}$ \\
\hline Sólidos totais (g/L) & $3,99 \pm 0,33^{\mathrm{a}}$ & $\begin{array}{c}2,27 \pm 0,23^{b} \\
(43,13)\end{array}$ & $\begin{array}{c}1,99 \pm 0,28^{\mathrm{b}} \\
(49,87)\end{array}$ & $\begin{array}{c}2,01 \pm 0,94^{\mathrm{b}} \\
(49,64)\end{array}$ \\
\hline Nitrito (mg/L) & $0,41 \pm 0,05^{\mathrm{a}}$ & $\begin{array}{c}0,096 \pm 0,05^{\mathrm{b}} \\
(76,57)\end{array}$ & $\begin{array}{c}0,06 \pm 0,008^{\mathrm{b}} \\
(84,21)\end{array}$ & $\begin{array}{c}0,06 \pm 0,03^{\mathrm{b}} \\
(83,97)\end{array}$ \\
\hline DQO (mg. $\left.\mathrm{L}^{-1}\right)$ & $1854,67 \pm 139,3^{a}$ & $\begin{array}{c}1199,83 \pm 157,0^{\mathrm{ab}} \\
(35.30)\end{array}$ & $\begin{array}{c}1089,5 \pm 384,9^{b} \\
(41,26)\end{array}$ & $\begin{array}{c}1089,33 \pm 370,5^{\mathrm{b}} \\
(41,27)\end{array}$ \\
\hline Cor (mg Pt/L) & $873 \pm 69,31^{a}$ & $\begin{array}{c}240,5 \pm 28,51^{\mathrm{b}} \\
(72,51)\end{array}$ & $\begin{array}{c}212,67 \pm 72,95^{\mathrm{bc}} \\
(75,64)\end{array}$ & $\begin{array}{c}77 \pm 10,58^{c} \\
(91,17)\end{array}$ \\
\hline
\end{tabular}

M1- membrana de 150 kDa; M2-membrana de 30 kDa; M3- membrana de $10 \mathrm{kDa}$

a,b,c Médias seguidas de letras diferentes, na linha, diferem estatisticamente entre si pelo teste de Tukey $(p<0,05)$. 


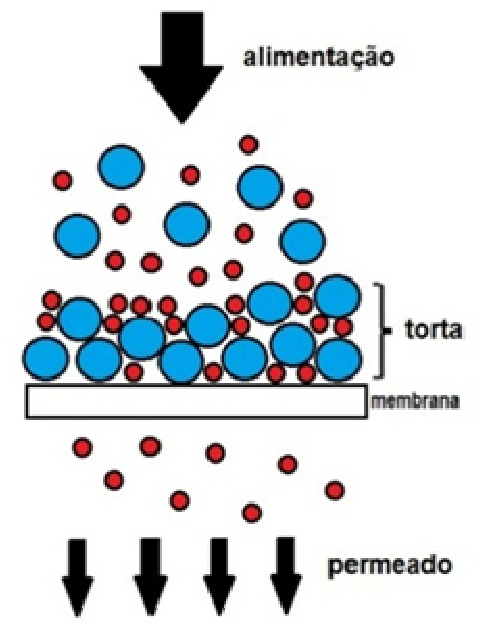

(a)

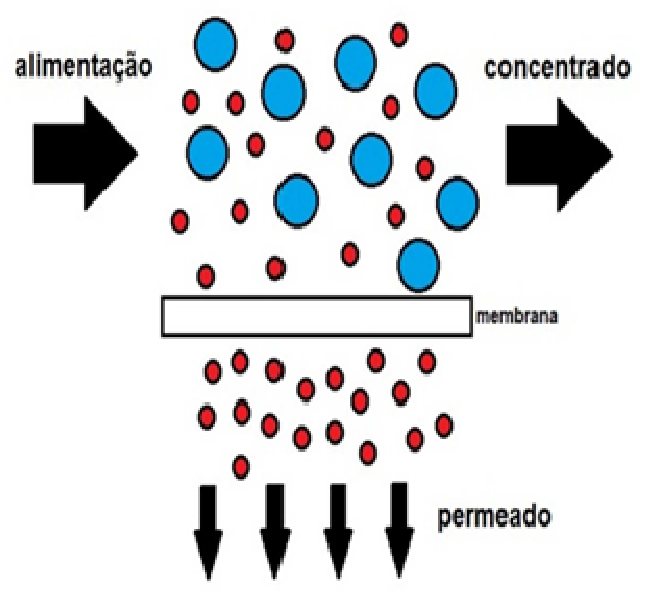

(b)

Figura 1. Tipos de filtração por membranas (a) frontal (b) tangencial. (Fonte: Ghiggi, 2011).

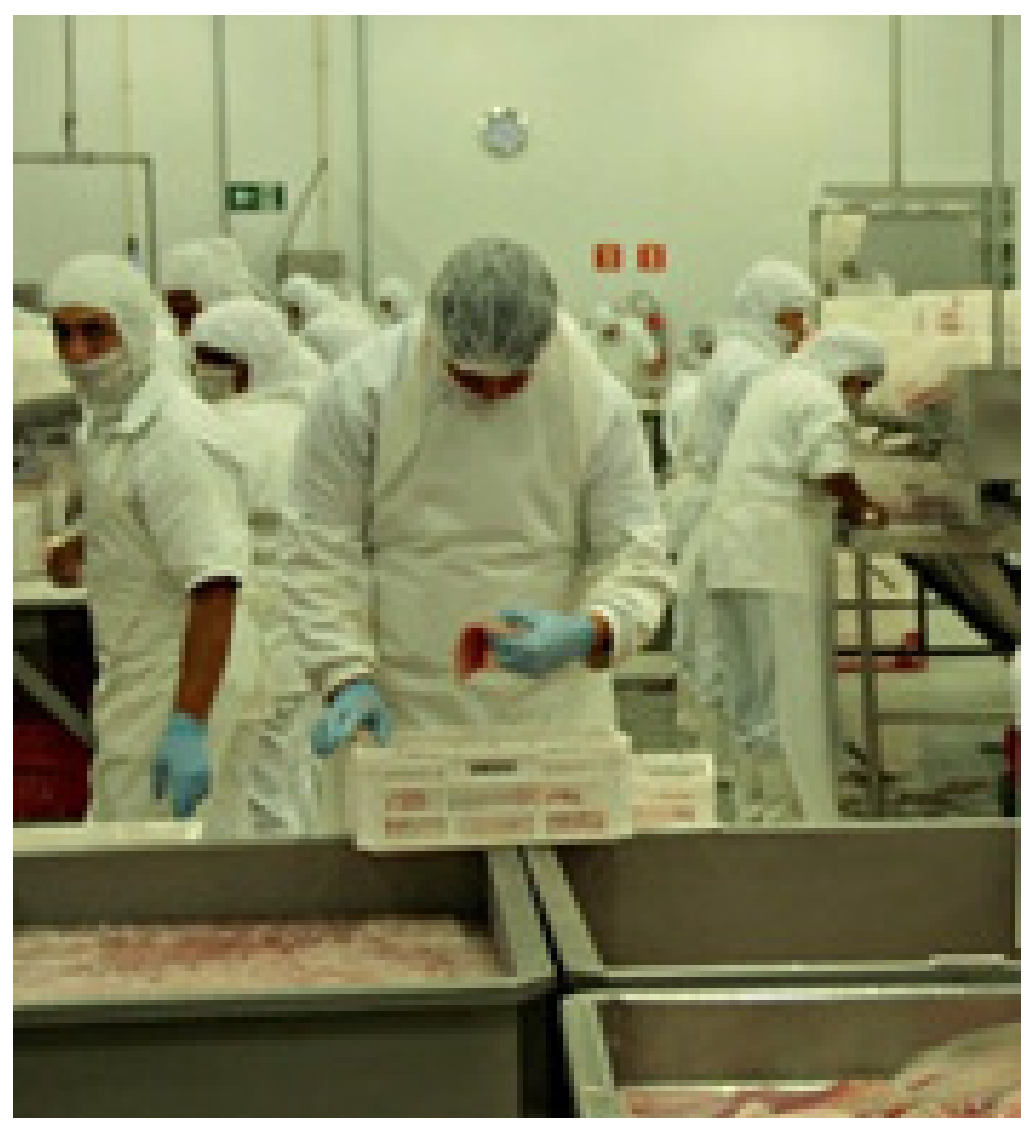

Figura 2. Vista do tanque de lavagem dos filés, de onde foi coletado o efluente utilizado nos experimentos. 


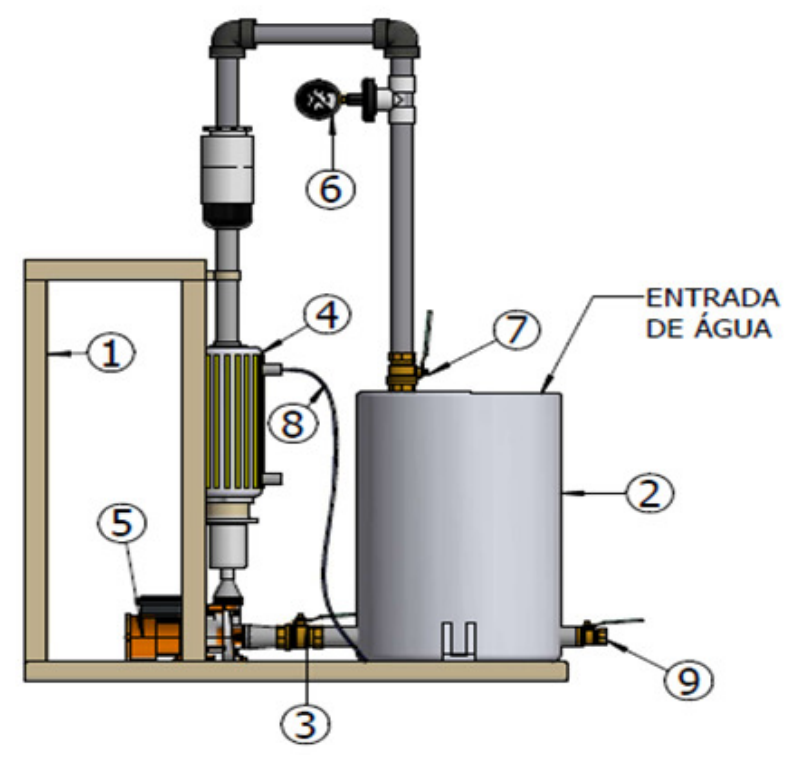

Figura 3. Esquema do sistema de filtração tangencial utilizado no experimento. 1) alça para movimentação; 2) tanque de alimentação; 3) válvula controladora de vazão 1; 4) membrana; 5) bomba de alimentação; 6) manômetro; 7) válvula controladora de vazão 2; 8) saída do permeado; 9) saída do retentado.

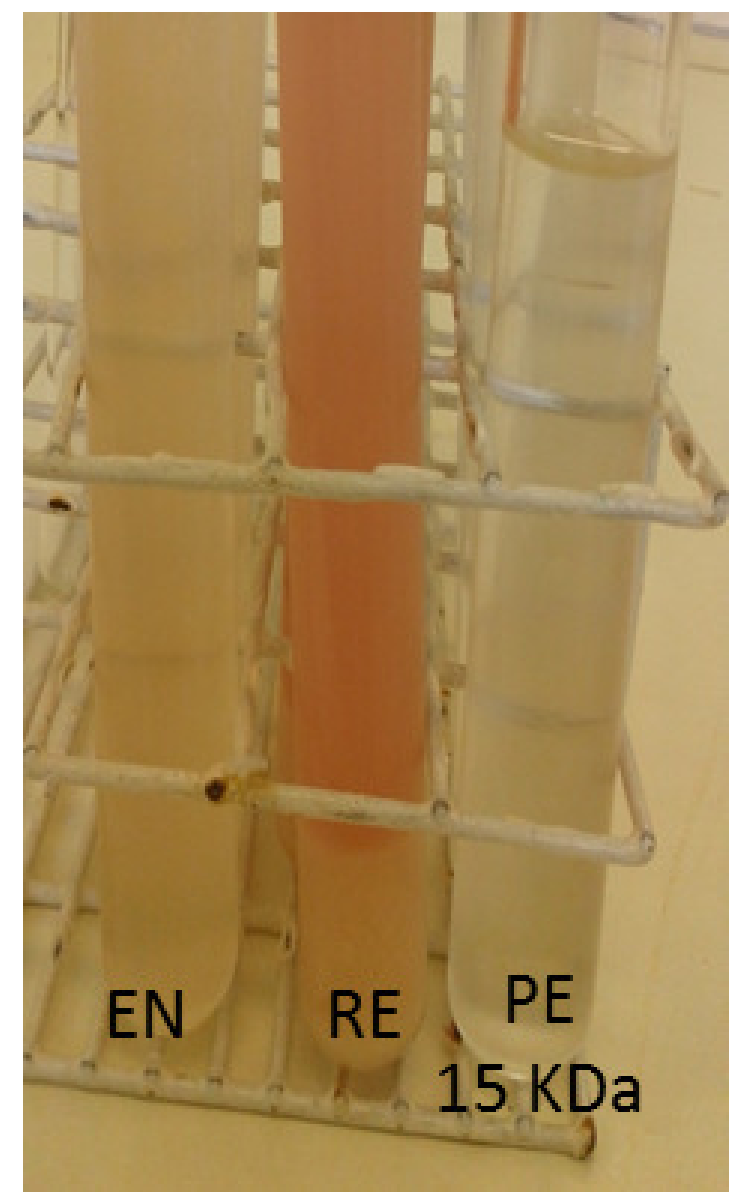

Figura 4. Aspectos do efluente antes e após a filtração tangencial com membrana de 15 kDa. EN: efluente in natura, não filtrado; RE: retentado obtido após a filtração; PE: permeado obtido após a filtração. 